\title{
On the dissipation rate of temperature fluctuations in stably stratified flows
}

\author{
Sukanta Basu' ${ }^{1}$. Adam W. DeMarco² $\cdot$ Ping $\mathrm{He}^{3}$
}

Received: 13 January 2020 / Accepted: 10 August 2020 / Published online: 25 August 2020

(c) The Author(s) 2020

\begin{abstract}
In this study, we explore several integral and outer length scales of turbulence which can be formulated by using the dissipation of temperature fluctuations $(\chi)$ and other relevant variables. Our analyses directly lead to simple yet non-trivial parameterizations for both spatially-averaged $\bar{\chi}$ and the structure parameter of temperature $\left(C_{T}^{2}\right)$. For our purposes, we make use of high-fidelity data from direct numerical simulations of stratified channel flows.
\end{abstract}

Keywords Integral length scale $\cdot$ Outer length scale $\cdot$ Ozmidov scale $\cdot$ Stable boundary layer $\cdot$ Structure parameter

\section{Introduction}

The molecular dissipation of temperature fluctuations $(\chi)$ is an important variable for characterizing turbulent mixing in various environmental flows. It is frequently used in micrometeorology (e.g., [66]) and atmospheric optics (e.g., [43]). Furthermore, any higher-order closure model requires solving a prognostic equation or a diagnostic parameterization for ensemble-averaged $\bar{\chi}$ (refer to $[15,36,40,65]$ ) .

Over the years, a number of studies focused on the correlation between turbulent kinetic energy dissipation rate $(\varepsilon)$ and $\chi$ (e.g., $[2,5-7,29,69])$. In addition, some papers reported on the probability density function, spatio-temporal intermittency and anomalous scaling of $\chi$ (e.g., $[6,52,54])$. Often, $\chi$ has been found to be more intermittent (commonly quantified by the multifractal scaling exponents) and non-Gaussian than $\varepsilon$ (e.g., [50, 54]).

Most of these previous studies primarily focused on the instantaneous, localized traits of the dissipation fields. Instead, we are interested to better quantify their spatially averaged characteristics. Towards this goal, we first investigate the statistical properties of several length scales which can be formulated based on $\bar{\chi}$ and other relevant variables. Based on

Sukanta Basu

s.basu@tudelft.nl

1 Faculty of Civil Engineering and Geosciences, Delft University of Technology, Delft, The Netherlands

2 United States Air Force, Washington, D.C., USA

3 Department of Aerospace Engineering, University of Michigan, Ann Arbor, USA 
these findings, we then derive simple parameterizations for $\bar{\chi}$ and temperature structure parameter $\left(C_{T}^{2}\right)$. For all the analyses, we utilize a direct numerical simulation (DNS) database of stably stratified flows which is discussed in the following section.

\section{Direct numerical simulation}

Recently, for the parameterization of optical turbulence, He and Basu [31] created a DNS database using a massively parallel DNS code, called HERCULES [30]. The DNS runs were conducted by solving the normalized Navier-Stokes and temperature equations, as shown in Eqs. 1-3 (using Einstein's summation notation):

$$
\begin{gathered}
\frac{\partial u_{n, i}}{\partial x_{n, i}}=0 \\
\frac{\partial u_{n, i}}{\partial t_{n}}+\frac{\partial u_{n, i} u_{n, j}}{\partial x_{n, j}}=-\frac{\partial p_{n}}{\partial x_{n, i}}+\frac{1}{R e_{b}} \frac{\partial}{\partial x_{n, j}} \frac{\partial u_{n, i}}{\partial x_{n, j}}+\Delta P \delta_{i 1}+R i_{b} \theta_{n} \delta_{i 3}, \\
\frac{\partial \theta_{n}}{\partial t_{n}}+\frac{\partial \theta_{n} u_{n, j}}{\partial x_{n, j}}=\frac{1}{\operatorname{Re} \operatorname{Pr}} \frac{\partial}{\partial x_{n, j}} \frac{\partial \theta_{n}}{\partial x_{n, j}}
\end{gathered}
$$

where $u_{n}$ and $x_{n}$ are the normalized velocity and coordinate vectors, respectively, with the subscript $i$ denoting the $i$ th vector component, $t_{n}$ is the normalized time, $p_{n}$ is the normalized pressure, $\Delta P$ is the streamwise pressure gradient driving the flow, and $\theta_{n}$ is the normalized potential temperature. The bulk Richardson number is denoted by:

$$
R i_{b}=\frac{\left(\Theta_{t o p}-\Theta_{b o t}\right) g h}{U_{b}^{2} \Theta_{t o p}},
$$

where $g$ denotes the gravitational acceleration, and $\Theta_{\text {top }}$ and $\Theta_{b o t}$ represent potential temperature at the top and the bottom of the channel, respectively. $\operatorname{Pr}=v / k=0.7$ is the Prandtl number with $k$ being the thermal diffusivity, and $R e_{b}=\frac{U_{b} h}{v}$ is the bulk Reynolds number with $h, U_{b}$, and $v$ being the channel height, the bulk (averaged) velocity in the channel, and the kinematic viscosity, respectively. The bulk Reynolds number was fixed at 20,000 for all the simulations.

The computational domain size for all the DNS runs was $L_{x} \times L_{y} \times L_{z}=18 h \times 10 h \times h$. The domain was discretized by $2304 \times 2048 \times 288$ grid points in streamwise, spanwise, and wall-normal directions, respectively. A total of five simulations were performed with gradual increase in the temperature difference between the top and bottom walls (effectively by increasing $R i_{b}$ ) to mimic the nighttime cooling of the land-surface. The normalized cooling rates $(C R), \partial R i_{b} / \partial T_{n}$, ranged from $1 \times 10^{-3}$ to $5 \times 10^{-3}$; where, $T_{n}$ is a nondimensional time $\left(=t U_{b} / h\right)$.

All the simulations used fully developed neutrally stratified flows $\left(R i_{b}=0\right)$ as initial conditions and evolved for up to $T_{n}=100$. The simulation results were output every 10 non-dimensional time. To avoid spin-up issues, in the present study, we only use data for the last five output files (i.e., $60 \leq T_{n} \leq 100$ ). Furthermore, we only consider data from the 
region $0.1 h \leq z \leq 0.5 h$ to discard any blocking effect of the surface or avoid any laminarization in the upper part of the open channel. Vertical profiles and some basic statistics from these simulations have been documented in "Appendix 3".

The mean dissipation of turbulent kinetic energy and temperature fluctuations are computed as follows:

$$
\begin{gathered}
\bar{\varepsilon}=v \overline{\left(\frac{\partial u_{i}^{\prime}}{\partial x_{j}} \frac{\partial u_{i}^{\prime}}{\partial x_{j}}\right)}, \\
\bar{\chi}=2 k \overline{\left(\frac{\partial \theta^{\prime}}{\partial x_{j}} \frac{\partial \theta^{\prime}}{\partial x_{j}}\right)} .
\end{gathered}
$$

In the above equations, and in the rest of the paper, the "overbar" notation is used to denote mean quantities. Horizontal (planar) averaging operation is performed for all the cases. The "prime" symbol is used to represent the fluctuation of a variable with respect to its planar averaged value.

In a recent paper, Basu et al. [10] utilized this DNS database to derive parameterizations for $\bar{\varepsilon}$. In the present work, the focus is placed on $\bar{\chi}$.

\section{Integral length scales}

From the DNS-generated data, we first calculate two different integral length scales as follows:

$$
\begin{gathered}
\mathcal{L} \equiv \frac{\bar{e}^{-3 / 2}}{\bar{\varepsilon}}, \\
\mathcal{L}_{\theta} \equiv \frac{e^{1 / 2} \sigma_{\theta}^{2}}{\bar{\chi}},
\end{gathered}
$$

where $\bar{e}$ and $\sigma_{\theta}^{2}$ denote turbulent kinetic energy (TKE) and the variance of temperature, respectively.

Based on the original ideas of Taylor [58], both [49, 59] provided a heuristic derivation of $\mathcal{L}$. Given TKE $(\bar{e})$ and mean energy dissipation rate $(\bar{\varepsilon})$, an associated integral time scale can be approximated as $\bar{e} / \bar{\varepsilon}$. One can further assume $\sqrt{\bar{e}}$ to be the corresponding velocity scale. Thus, an integral length scale can be approximated as $\bar{e}^{3 / 2} / \bar{\varepsilon}$. Using dimensional arguments, an analogous length scale $\mathcal{L}_{\theta}$ can be formulated based on temperature fluctuations $[1,68]$.

In the top-panels of Fig. 1, normalized values of $\mathcal{L}$ and $\mathcal{L}_{\theta}$ are plotted against the gradient Richardson number $\left(R i_{g}=N^{2} / S^{2}\right)$; where, $N$ is the Brunt-Väisäla frequency and $S$ is the magnitude of wind shear. In these plots, we have marked four specific points based on the data from DNS run with imposed cooling rate of $10^{-3}$ to better understand the effects of height and stability on the integral length scales. The points $p_{1}$ and $p_{2}$ represent data from $z / h=0.1$ and $z / h=0.5$, respectively at non-dimensional time $\left(T_{n}\right)$ of 60 . Similarly, $q_{1}$ and 

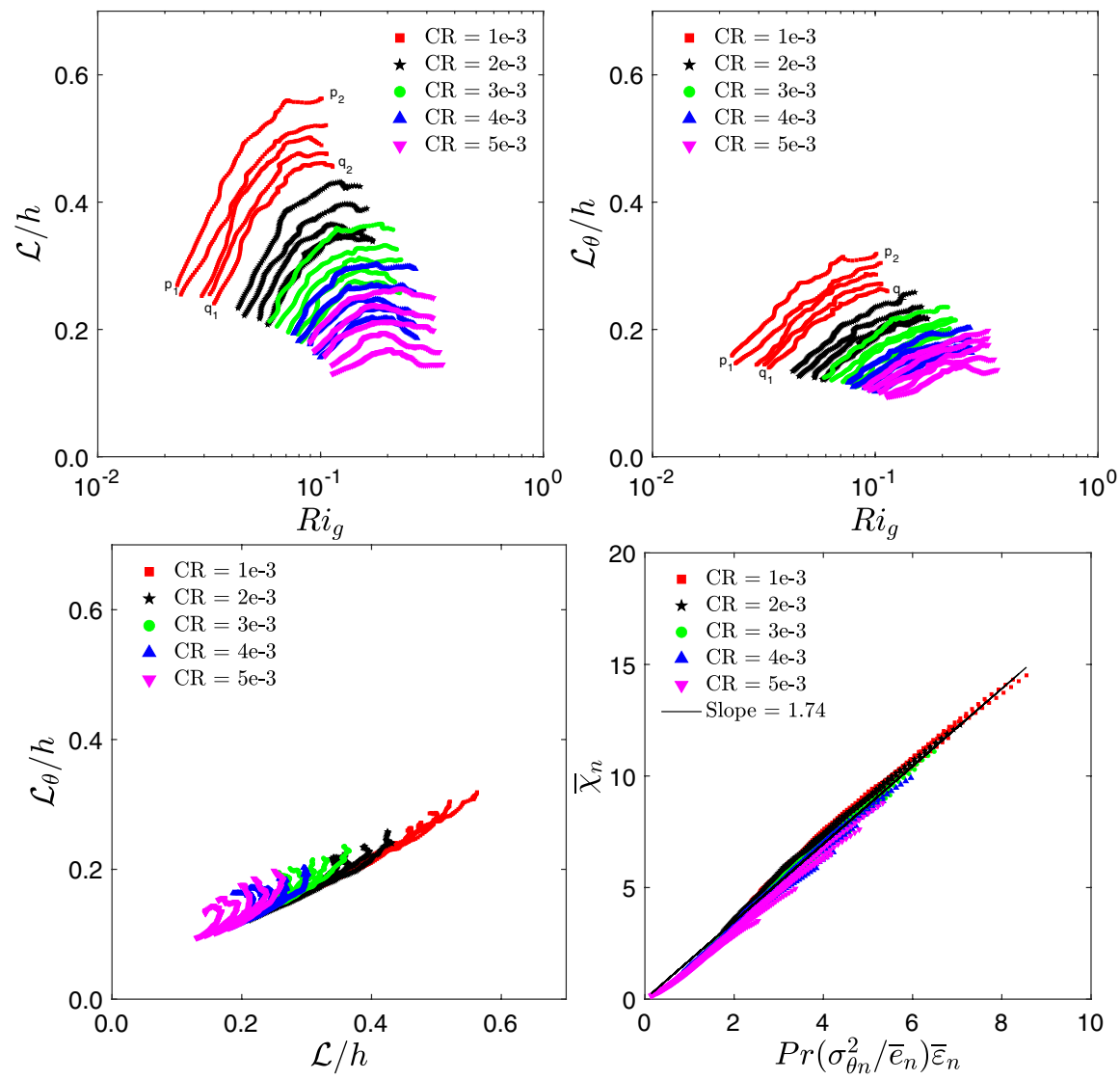

Fig. 1 Top panel: integral length scales as functions of gradient Richardson number. Both the length scales are normalized by the height of the open channel $(h)$. Bottom-left panel: scatter plot of $\mathcal{L}$ versus $\mathcal{L}_{\theta}$. Bottom-right panel: normalized $\bar{\chi}$ as a function of normalized $\bar{\varepsilon}, \bar{e}$, and $\sigma_{\theta}^{2}$. Please refer to Eq. 7. Simulated data from five different DNS runs are represented by different colored symbols in these plots. In the legends, $C R$ represents normalized cooling rates

$q_{2}$ are associated with data from $z / h=0.1$ and $z / h=0.5$, respectively at non-dimensional time $\left(T_{n}\right)$ of 100 .

Physically, one would expect the integral scales to be increasing with height as long as the eddies feel the presence of the surface (near-neutral or weakly stable condition). For very stable conditions, the eddies no longer feel the presence of the surface. In the atmospheric boundary layer literature, such a situation is known as the z-less condition $[28,64]$. Under the influence of strong stability, the integral length scales become moreor-less independent of the height above the surface.

From Fig. 1, it is clear that the integral length scales increase with height and they slowly decrease with time in all the simulations due to the increasing stability effects. Simulations with higher cooling rates have smaller integral length scales. Some of these runs (e.g., $C R=5 \times 10^{-3}$ ) exhibit z-less behavior due to strong stability effects. 
Given the similar trends of normalized $\mathcal{L}$ and $\mathcal{L}_{\theta}$, they are plotted against each other in the bottom-left panel of Fig. 1. There is (approximately) a linear relationship between these length scales. If $\mathcal{L} \propto \mathcal{L}_{\theta}$, it is straightforward to derive from Eqs. 6:

$$
\frac{\bar{\chi}}{\bar{\varepsilon}} \propto \frac{\sigma_{\theta}^{2}}{\bar{e}} .
$$

This relationship was first reported by Béguier et al. [11] for shear flow turbulence. In a follow-up study, Elghobashi and Launder [22] hypothesized that the similarity of the generation processes of TKE and scalar variance is at the root of this intriguing relationship. In contrast to shear flows, they did not find Eq. 7 to hold for thermal mixed layer.

In the bottom-right panel of Fig. 1, we demonstrate the approximate validity of Eq. 7. Linear least-square regression with bootstrapping [21, 42] is used to estimate the slope of the fitted line. Given that the collapse of the data points is quite reasonable, the relationship $\bar{\chi}=1.74 \bar{\varepsilon} \frac{\sigma_{\theta}^{2}}{\bar{e}}$ might be useful for practical applications.

Please note that the appearance of the Prandtl number $(P r)$ in Fig. 1 (bottom-right panel) is due to the normalization of variables in DNS; "Appendix 2" provides further details. Throughout the paper, the subscript " $n$ " is used to denote a normalized variable.

\section{Outer length scales}

Both shear and buoyancy prefer to deform larger eddies compared to smaller ones [16, 34, $39,53]$. Turbulent eddies are not affected by shear and buoyancy if they are smaller than the outer length scales (OLSs). Ozmidov $\left(L_{O Z}\right)$ and Corrsin $\left(L_{C}\right)$ length scales are the most commonly used OLSs in the literature. They are defined as [17, 20, 47]:

$$
\begin{aligned}
L_{O Z} & \equiv\left(\frac{\bar{\varepsilon}}{N^{3}}\right)^{1 / 2}, \\
L_{C} & \equiv\left(\frac{\bar{\varepsilon}}{S^{3}}\right)^{1 / 2} .
\end{aligned}
$$

Eddies which are smaller than $L_{O Z}$ are not affected by buoyancy; similarly, shear does not influence the eddies of size less than $L_{C}$. In other words, the eddies can be assumed to be isotropic if they are smaller than both $L_{O Z}$ and $L_{C}$.

Since $\mathcal{L}$ changes across the simulations, the OLS values are normalized by corresponding $\mathcal{L}$ values and plotted as functions of $R i_{g}$ in Fig. 2. The collapse of the data from different runs, on to seemingly universal curves, is remarkable for all the cases except for $R i_{g}>0.2$. We would like to mention that similar scaling behavior was not found if other normalization factors (e.g., $h$ ) are used.

Normalized $L_{O Z}$ decreases monotonically with $R i_{g}$. In contrast, normalized $L_{C}$ barely exhibits any sensitivity to $R i_{g}$ (except for $R i_{g}>0.1$ ). Even for weakly-stable condition, it is less than $20 \%$ of $\mathcal{L}$. Based on the expressions of $L_{O Z}, L_{C}$ and $R i_{g}$, we can write:

$$
\frac{L_{C}}{L_{O Z}}=\left(\frac{N}{S}\right)^{3 / 2}=R i_{g}^{3 / 4} .
$$



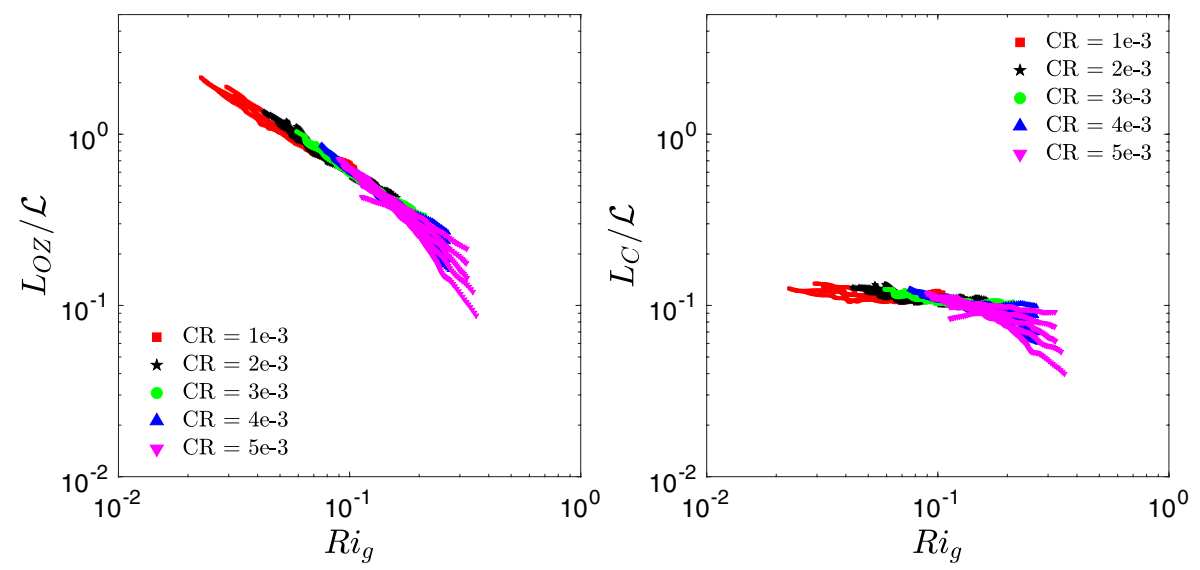

Fig. 2 Ozmidov (left panel) and Corrsin (right panel) length scales as functions of gradient Richardson numbers. These length scales are normalized by the integral length scale $(\mathcal{L})$. Simulated data from five different DNS runs are represented by different colored symbols in these plots. In the legends, $C R$ represents normalized cooling rates

Thus, for $R i_{g}<1$, one expects $L_{C}<L_{O Z}$; this relationship is fully supported by Fig. 2. In comparison to the buoyancy effects, the shear effects are felt at smaller length scales for the entire stability range considered in the present study.

Dissipation rate of turbulent kinetic energy is used in the definitions for both $L_{O Z}$ and $L_{C}$. However, it is also possible to formulate OLSs based on the dissipation rate of temperature fluctuations as follows:

$$
\begin{gathered}
L_{1} \equiv\left(\frac{g}{\Theta_{0}}\right)^{-1 / 4} \bar{\chi}^{1 / 2}\left(\frac{\partial \bar{\theta}}{\partial z}\right)^{-5 / 4}, \\
L_{2} \equiv \bar{\varepsilon}^{-1 / 4} \bar{\chi}^{3 / 4}\left(\frac{\partial \bar{\theta}}{\partial z}\right)^{-3 / 2}, \\
L_{3} \equiv \bar{\chi}^{1 / 2}\left(\frac{\partial \bar{\theta}}{\partial z}\right)^{-1} S^{-1 / 2}, \\
L_{4} \equiv\left(\frac{g}{\Theta_{0}}\right) \bar{\chi}^{1 / 2} S^{-5 / 2},
\end{gathered}
$$

where $(\partial \bar{\theta} / \partial z)$ is the vertical gradient of mean potential temperature and $\Theta_{0}$ is a reference potential temperature. These length scales were proposed by Panchev based on dimensional analysis [41, 48]. Characteristics of yet another OLS proposed by Bolgiano [13, 14] and Obukhov [46] is discussed separately in "Appendix 1".

In Fig. 3 , the $\bar{\chi}$-based length scale formulations are plotted against $R i_{g}$. Similar to $L_{O Z}$, the normalized $L_{1}$ monotonically decrease with increasing $R i_{g}$. Whereas, the normalized $L_{4}$ increase with $R i_{g}$ in an unphysical manner. It is quite evident that both the normalized $L_{2}$ and $L_{3}$ scales behave very similar to $L_{C}$ (see right panel of Fig. 2). 

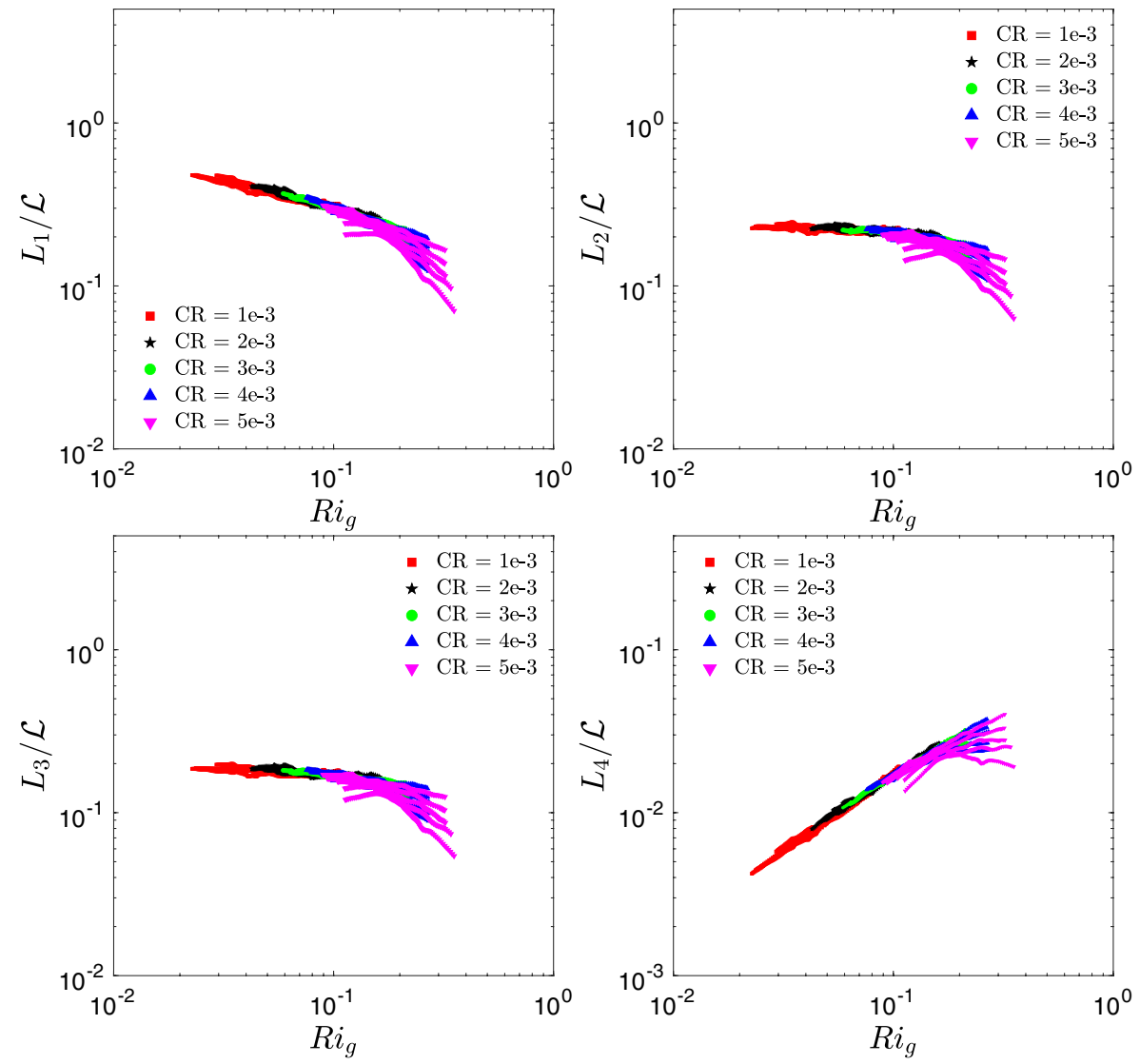

Fig. $3 \chi$-based length scales as functions of gradient Richardson numbers. These length scales are normalized by the integral length scale. Simulated data from five different DNS runs are represented by different colored symbols in these plots. In the legends, $C R$ represents normalized cooling rates

Given the trends in Fig. 3, we plotted a few inter-relationships of OLSs in Fig. 4. In each case, the collapse of DNS-based data on a single curve is excellent. In the case of $L_{1}$-versus- $L_{O Z}$ plot, the curve is nonlinear. However, in the case of $L_{2}$-versus- $L_{C}$ and $L_{3}$-versus- $L_{C}$ plots, the data fall on more-or-less straight lines. The regressed slopes are reported in the legends of these plots.

If we assume $L_{2} \equiv L_{C}$, based on Eq. 8 b and Eq. 10b, it is trivial to arrive at:

$$
\frac{\bar{\chi}}{\overline{\bar{\varepsilon}}} \approx \frac{\left(\frac{\partial \bar{\theta}}{\partial z}\right)^{2}}{S^{2}} .
$$

Interestingly, the assumption of $L_{3} \equiv L_{C}$ also leads to the same equation. As a matter of fact, this equation can be derived from the budget equations of TKE and temperature variance with certain assumptions as elaborated below. Assuming steady-state condition, horizontal homogeneity, and neglecting the secondary terms (e.g., transport), we can write: 

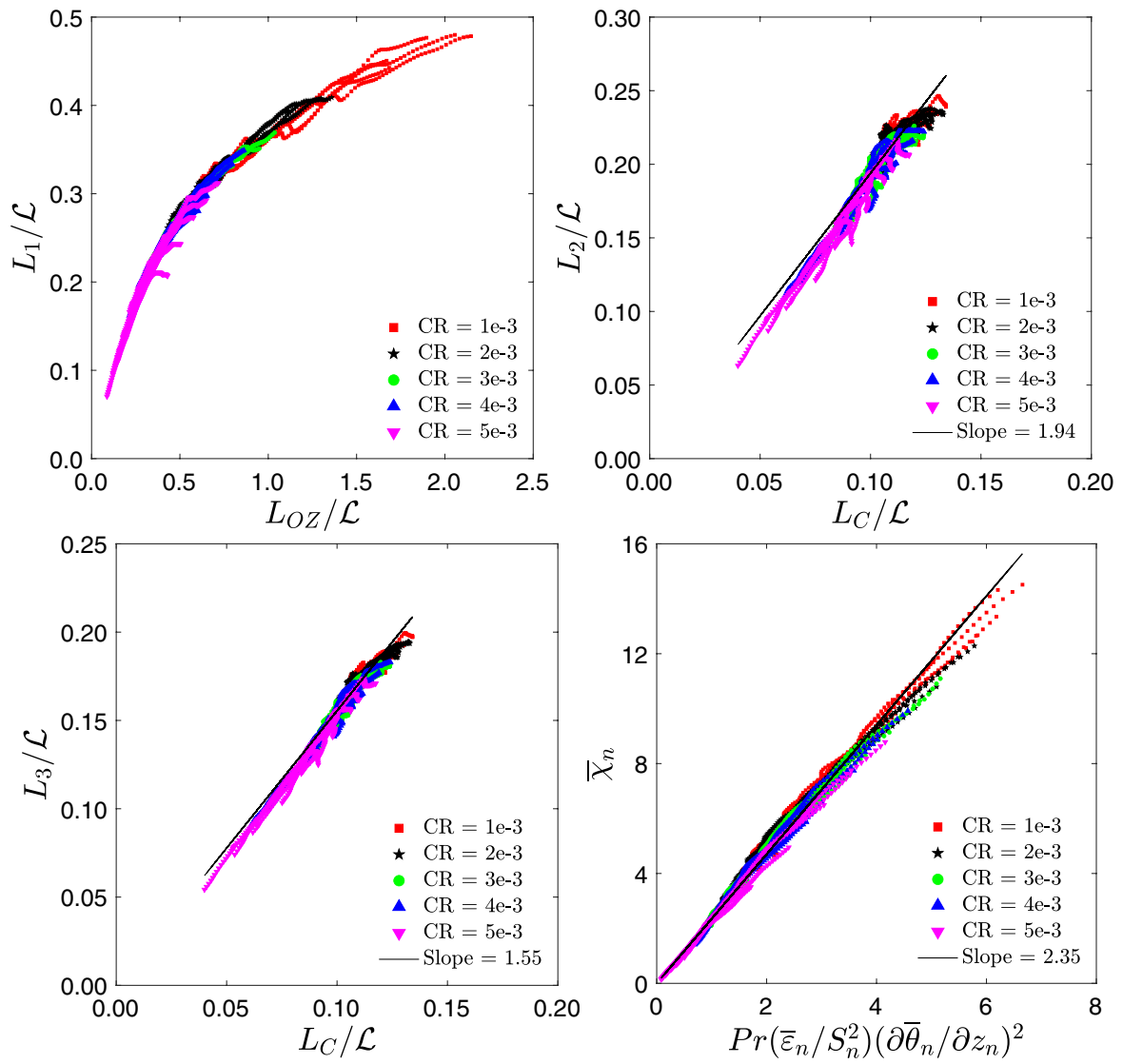

Fig. 4 Variation of the normalized $\bar{\chi}$-based length scales against the normalized Ozmidov length scale (topleft panel) and the normalized Corrsin length scale (top-right and bottom-left panels). Bottom-right panel: normalized $\bar{\chi}$ as a function of normalized $\bar{\varepsilon}, S$, and $(\partial \bar{\theta} / \partial z)$. Please refer to Eq. 11 . Simulated data from five different DNS runs are represented by different colored symbols in these plots. In the legends, $C R$ represents normalized cooling rates

$$
\bar{\varepsilon}=-\overline{u^{\prime} w^{\prime}}\left(\frac{\partial \bar{u}}{\partial z}\right)-\overline{v^{\prime} w^{\prime}}\left(\frac{\partial \bar{v}}{\partial z}\right)+\left(\frac{g}{\Theta_{0}}\right) \overline{w^{\prime} \theta^{\prime}}
$$

and

$$
\bar{\chi}=-2 \overline{w^{\prime} \theta^{\prime}}\left(\frac{\partial \bar{\theta}}{\partial z}\right) .
$$

If we apply K-theory, these equations can be further simplified to:

$$
\bar{\varepsilon}=K_{M} S^{2}-K_{H}\left(\frac{g}{\Theta_{0}}\right)\left(\frac{\partial \bar{\theta}}{\partial z}\right),
$$

and 


$$
\bar{\chi}=2 K_{H}\left(\frac{\partial \bar{\theta}}{\partial z}\right)^{2},
$$

where $K_{M}$ and $K_{H}$ are eddy viscosity and diffusivity, respectively. By utilizing the definitions of $R i_{g}$ and turbulent Prandtl number $\left(P r_{T}=K_{M} / K_{H}\right)$, we can deduce from Eq. 13a and Eq. 13b:

$$
\frac{\bar{\chi}}{\bar{\varepsilon}}=\frac{2}{\left(P r_{T}-R i_{g}\right)} \frac{\left(\frac{\partial \bar{\theta}}{\partial z}\right)^{2}}{S^{2}} .
$$

Anderson [4] conducted a rigorous statistical analysis of the observational data collected at the British Antarctic Survey's Halley station on the the Antarctic. He avoided the selfcorrelation issue and proposed the following empirical relationship for $0.01<R i_{g}<0.25$ :

$$
\operatorname{Pr}_{T}^{-1}=(0.84 \pm 0.03) R i_{g}^{-0.105 \pm 0.012}
$$

Clearly, the $R i_{g}$-dependence of the Prandtl number is rather weak for small values of $R i_{g}$. Similar findings were reported in other experimental and modeling studies (e.g., [35, 38, 55]).

In the bottom-right panel of Fig. 4, we have plotted Eq. 11 in a normalized form. The slope of the fitted line is 2.35 . For $R i_{g}=0.2$, according to Eq. $15, P r_{T} \approx 1$. Thus, the ratio $2 /\left(P r_{T}-R i_{g}\right)$ is approximately 2.48. When $R i_{g}$ equals to $0.1, P r_{T} \approx 0.93$ following Eq. 15 . In this case, the ratio $2 /\left(P r_{T}-R i_{g}\right)$ is close to 2.40 . These values are not far from the estimated slope of 2.35 in Fig. 4 (bottom-right panel). In other words, our DNS-based results are in-line with past observational studies.

\section{Structure parameter of temperature $\left(C_{T}^{2}\right)$}

Using the DNS database of the current study, Basu et al. [10] recently found that $\bar{\varepsilon}=0.23 \bar{e} S$ and $\bar{\varepsilon}=0.63 \sigma_{w}^{2} S$ for $0<R i_{g}<0.2$. If we insert these formulations in Eq. 11 , we get:

$$
\bar{\chi} \approx\left(\frac{\bar{e}}{S}\right)\left(\frac{\partial \bar{\theta}}{\partial z}\right)^{2},
$$

and

$$
\bar{\chi} \approx\left(\frac{\sigma_{w}^{2}}{S}\right)\left(\frac{\partial \bar{\theta}}{\partial z}\right)^{2}
$$

The top panels of Fig. 5 strongly support the validity of these formulations. The proportionality constants in these equations are found to be equal to 0.55 and 1.47 , respectively.

By definition, $C_{T}^{2} \approx \bar{\varepsilon}^{-1 / 3} \bar{\chi}$. The proportionality constant is usually taken equal to 1.6 $[31,67]$. Thus, we can write: 

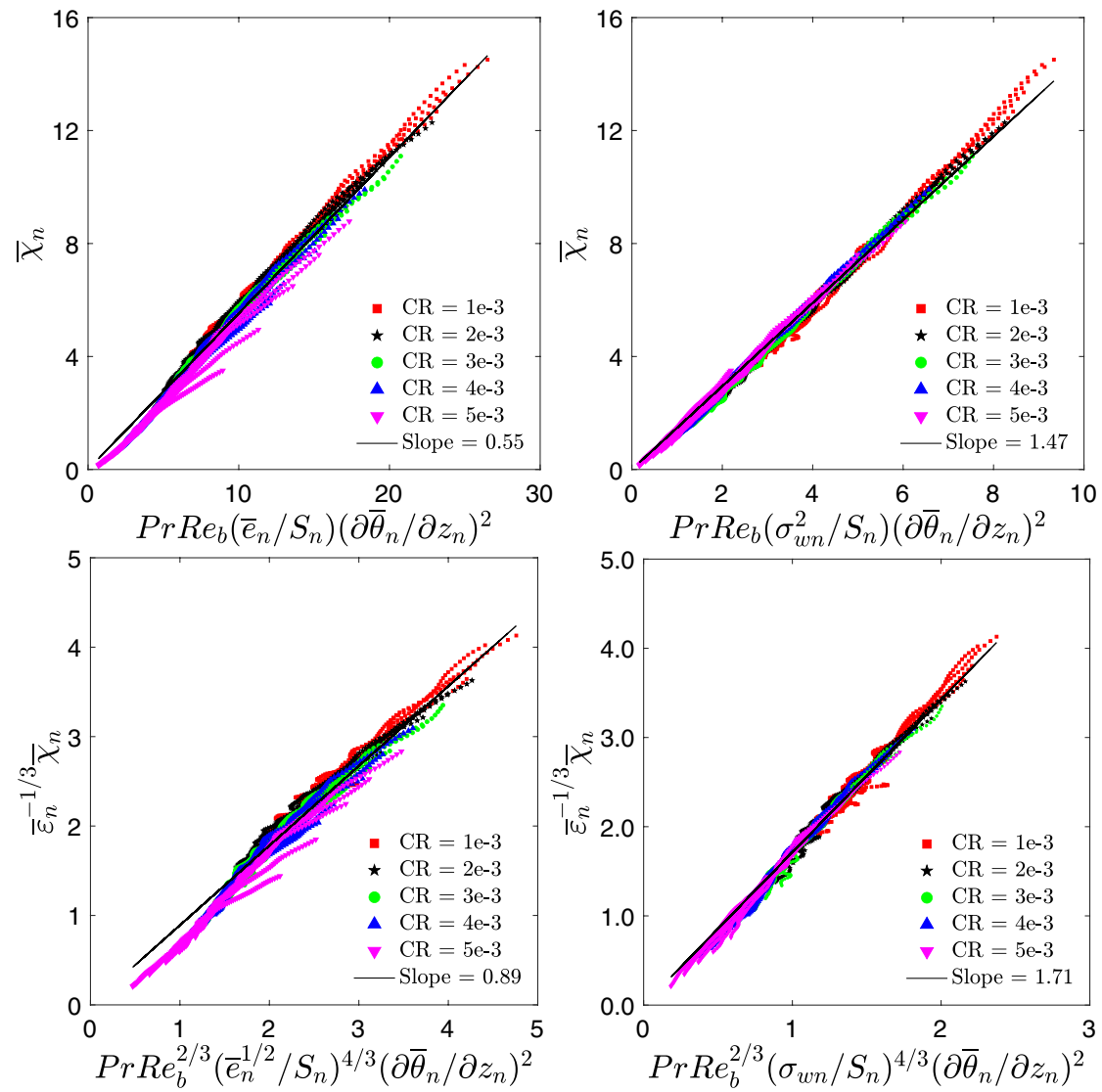

Fig. 5 Top panels: normalized $\bar{\chi}$ as a function of normalized $\bar{e}, \sigma_{w}^{2}, S$, and $(\partial \bar{\theta} / \partial z)$. Please refer to Eqs. 16 . Bottom panels: normalized $\bar{\varepsilon}^{-1 / 3} \bar{\chi}$ as a function of normalized $\bar{e}, \sigma_{w}^{2}, S$, and $(\partial \bar{\theta} / \partial z)$. Please refer to Eqs. 17. Simulated data from five different DNS runs are represented by different colored symbols in these plots. In the legends, $C R$ represents normalized cooling rates

$$
C_{T}^{2} \approx \bar{\varepsilon}^{-1 / 3} \bar{\chi} \approx\left(\frac{\bar{e}^{-1 / 2}}{S}\right)^{4 / 3}\left(\frac{\partial \bar{\theta}}{\partial z}\right)^{2},
$$

and

$$
C_{T}^{2} \approx \bar{\varepsilon}^{-1 / 3} \bar{\chi} \approx\left(\frac{\sigma_{w}}{S}\right)^{4 / 3}\left(\frac{\partial \bar{\theta}}{\partial z}\right)^{2} .
$$

In the bottom panels of Fig. 5, we establish that these equations (especially Eq. 17b) nicely hold for our DNS-generated data.

Based on theoretical and numerical work, Hunt et al. [32,33] proposed the shear-based length scales, $L_{H} \equiv\left(\frac{\bar{e}^{1 / 2}}{S}\right)$ and $L_{H} \equiv\left(\frac{\sigma_{w}}{S}\right)$, as the characteristic length scales for $0<R i_{g}<0.5$. Thus, we can re-write Eqs. 17 as: 


$$
C_{T}^{2} \approx L_{H}^{4 / 3}\left(\frac{\partial \bar{\theta}}{\partial z}\right)^{2} .
$$

A very similar equation was proposed by Tatarskii more than 50 years ago $[56,57]$, albeit with an OLS which needs to be prescribed. In the literature, several empirical parameterizations were proposed for this unknown length scale [8, 18, 19, 62]. In this study, based on DNS-generated data, we demonstrate that the outer length scale in Tatarskii's equation should be equal to $L_{H}$ for $0<R i_{g}<0.2$.

At this point, we point out an interesting relationship that one can further deduce from our findings. If we compare Eq. 7 against Eq. 11, we get:

$$
\frac{\sigma_{\theta}^{2}}{\bar{e}} \approx \frac{\left(\frac{\partial \bar{\theta}}{\partial z}\right)^{2}}{S^{2}} .
$$

Equivalently, one can write:

$$
\frac{\sigma_{\theta}}{\left(\frac{\partial \bar{\theta}}{\partial z}\right)} \approx \frac{\bar{e}^{1 / 2}}{S},
$$

or,

$$
L_{E} \equiv L_{H}
$$

where $L_{E}$ is a length scale proposed by Ellison [23]. The dependence of $L_{E}$ on $R i_{g}$ is documented in the left panel of Fig. 6. In the right panel, we show the one-to-one relationship between $L_{E}$ and $L_{H}$. With the exception of a few data points from the simulation with the strongest cooling rate, it is clear that these length scales are linearly related to each other. Thus, the following equation can be used as a viable alternative to Eq. 18:
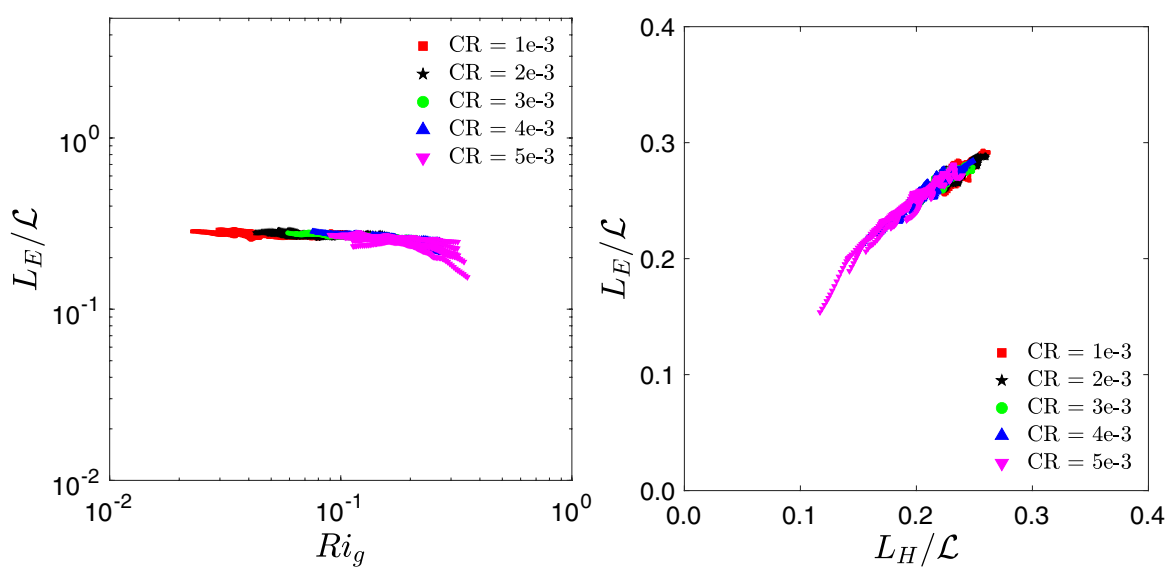

Fig. 6 Left panel: normalized Ellison length scale as a function of gradient Richardson number. Right panel: normalized Ellison length scale as a function of normalized Hunt length scale. Simulated data from five different DNS runs are represented by different colored symbols in these plots. In the legends, $C R$ represents normalized cooling rates 


$$
C_{T}^{2} \approx L_{E}^{4 / 3}\left(\frac{\partial \bar{\theta}}{\partial z}\right)^{2}
$$

In the literature, several studies have demonstrated the similarities between the so-called Thorpe scale $\left(L_{T} ;[60,61]\right)$ and $L_{E}$ using observed and simulated data (e.g., [34, 39]). A simple heuristic derivation was also provided by Gavrilov et al. [27]. Thus, it is plausible to replace $L_{E}$ with $L_{T}$ in Eq. 21:

$$
C_{T}^{2} \approx L_{T}^{4 / 3}\left(\frac{\partial \bar{\theta}}{\partial z}\right)^{2} .
$$

This equation was proposed by Basu [8] and was validated using observational data from a field campaign over Mauna Kea, Hawaii.

In summary, we conjecture that Eqs. 18, 21, and 22 are all valid parameterizations for $C_{T}^{2}$ as long as $R i_{g}$ does not exceed 0.2 . For larger values of $R i_{g}$, a different length scale might be more appropriate; our present DNS runs cannot shed light on such a strong stability regime.

\section{Concluding remarks}

In this study, we analyze DNS-generated data to characterize several integral and outer length scales. From these results, we propose simple parameterizations for $\chi$ and $C_{T}^{2}$ when gradient Richardson number is less than 0.2 . In the continuously turbulent atmospheric stable boundary layer, $R i_{g}$ is usually less than $0.2[26,45]$. Thus, the proposed parameterizations should be suitable for certain practical boundary layer problems. However, they will have limited applications for intermittently stable conditions.

In closing, we would like to emphasize the importance of Eq. 19. To the best of our knowledge, it was first reported by Fulachier and Dumas [25] from boundary layer experiments over a slightly heated plate. In a latter study, Fulachier and Antonia [24] found this formulation to hold for various other types of flows. They even concluded:

It seems therefore reasonable, from both mathematical and physical points of view, to seek a relationship, not between momentum and heat fluxes, as in the case with the Reynolds analogy, but preferably between the turbulent kinetic energy and the temperature variance.

To the best of our knowledge, Eq. 19 is not used in atmospheric boundary layer studies. Since our findings are in agreement, we strongly endorse the assertion of Fulachier and Antonia [24] and advocate further research on this equation.

Acknowledgements The first author thanks Bert Holtslag for having interesting discussion on this topic. The authors acknowledge computational resources obtained from the Department of Defense Supercomputing Resource Center (DSRC) for the direct numerical simulations. The views expressed in this paper do not reflect official policy or position by the US Air Force or the US Government.

Data and code availability The DNS code (HERCULES) is available from: https://github.com/friedenhe/ HERCULES. All the analysis codes and processed data are publicly available at https://doi.org/10.5281/ zenodo.3992818. Given the sheer size of the raw DNS dataset, it is not uploaded on to any repository; however, it is available upon request from the authors. 
Open Access This article is licensed under a Creative Commons Attribution 4.0 International License, which permits use, sharing, adaptation, distribution and reproduction in any medium or format, as long as you give appropriate credit to the original author(s) and the source, provide a link to the Creative Commons licence, and indicate if changes were made. The images or other third party material in this article are included in the article's Creative Commons licence, unless indicated otherwise in a credit line to the material. If material is not included in the article's Creative Commons licence and your intended use is not permitted by statutory regulation or exceeds the permitted use, you will need to obtain permission directly from the copyright holder. To view a copy of this licence, visit http://creativecommons.org/licenses/by/4.0/.

\section{Appendix 1: Bolgiano-Obukhov length scale}

Bolgiano [13, 14] and Obukhov [46] independently proposed a buoyancy-range scaling and the following OLS based on theoretical arguments:

$$
L_{B O} \equiv\left(\frac{g}{\Theta_{0}}\right)^{-3 / 2} \bar{\varepsilon}^{5 / 4} \bar{\chi}^{-3 / 4} .
$$

Several laboratory and numerical studies (e.g., [12, 44]) reported the existence of Bolgiano-Obukhov scaling in unstable condition. However, studies involving stably stratified conditions are rather limited [3, 51]. Recently, [37, 63] reported that the Bolgiano-Obukhov scaling only exists for moderately stable condition. It is non-existent for near-neutral and very stable conditions.

In Fig. 7, we show the traits of $L_{B O}$ as a function of $R i_{g}$. Similar to $L_{O Z}$ and $L_{1}$, this length scale also shows a decreasing trend with increasing stability. However, the relationship between $L_{B O}$ and $L_{O Z}$ is nonlinear. As a consequence, we were unable to derive any simple formulation involving $\bar{\varepsilon}, \bar{\chi}$, and other variables.
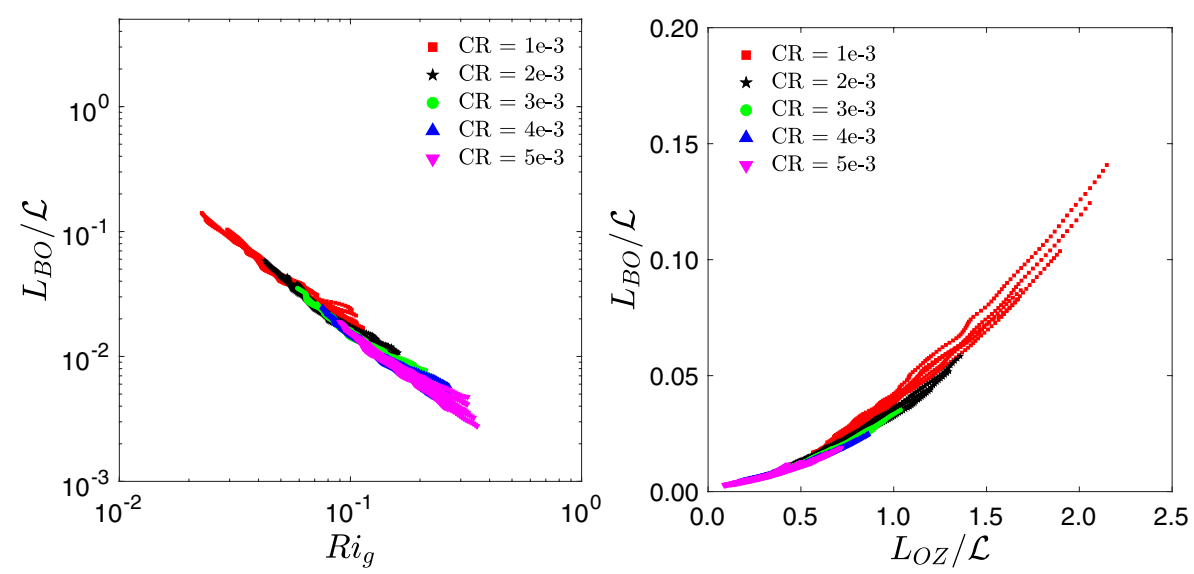

Fig. 7 Left panel: normalized Bolgiano length scale as a function of gradient Richardson number. Right panel: normalized Bolgiano length scale as a function of normalized Ozmidov length scale. Simulated data from five different DNS runs are represented by different colored symbols in these plots. In the legends, $C R$ represents normalized cooling rates 


\section{Appendix 2: Normalization of DNS variables}

In DNS, the relevant variables are normalized as follows:

$$
\begin{gathered}
z_{n}=\frac{z}{h}, \\
u_{n}=\frac{u}{U_{b}}, \\
v_{n}=\frac{v}{U_{b}}, \\
w_{n}=\frac{w}{U_{b}}, \\
\theta_{n}=\frac{\theta-\Theta_{t o p}}{\Theta_{t o p}-\Theta_{b o t}} .
\end{gathered}
$$

After differentiation, we get:

$$
\begin{gathered}
\frac{\partial u}{\partial z}=\frac{\partial u}{\partial z_{n}} \frac{\partial z_{n}}{\partial z}=\frac{\partial u}{\partial u_{n}} \frac{\partial u_{n}}{\partial z_{n}} \frac{\partial z_{n}}{\partial z}=\frac{U_{b}}{h} \frac{\partial u_{n}}{\partial z_{n}}, \\
\frac{\partial v}{\partial z}=\frac{\partial v}{\partial z_{n}} \frac{\partial z_{n}}{\partial z}=\frac{\partial v}{\partial v_{n}} \frac{\partial v_{n}}{\partial z_{n}} \frac{\partial z_{n}}{\partial z}=\frac{U_{b}}{h} \frac{\partial v_{n}}{\partial z_{n}}, \\
S=\sqrt{\left(\frac{\partial \bar{u}}{\partial z}\right)^{2}+\left(\frac{\partial \bar{v}}{\partial z}\right)^{2}}=\frac{U_{b}}{h} S_{n}, \\
\frac{\partial \theta}{\partial z}=\frac{\partial \theta}{\partial z_{n}} \frac{\partial z_{n}}{\partial z}=\frac{\partial \theta}{\partial \theta_{n}} \frac{\partial \theta_{n}}{\partial z_{n}} \frac{\partial z_{n}}{\partial z}=\left(\frac{\Theta_{t o p}-\Theta_{b o t}}{h}\right) \frac{\partial \theta_{n}}{\partial z_{n}} .
\end{gathered}
$$

The gradient Richardson number can be expanded as:

$$
R i_{g}=\frac{N^{2}}{S^{2}}=\frac{\left(\frac{g}{\Theta_{0}}\right)\left(\frac{\partial \bar{\theta}}{\partial z}\right)}{S^{2}}=\left(\frac{g}{\Theta_{t o p}}\right)\left(\frac{\Theta_{t o p}-\Theta_{b o t}}{h}\right)\left(\frac{h}{U_{b}}\right)^{2} \frac{\left(\frac{\partial \bar{\theta}_{n}}{\partial z_{n}}\right)}{S_{n}^{2}} .
$$

Using the definition of $R i_{b}$ (see Sect. 2), we re-write $R i_{g}$ as follows:

$$
R i_{g}=R i_{b} \frac{\left(\frac{\partial \bar{\theta}_{n}}{\partial z_{n}}\right)}{S_{n}^{2}}
$$

Similarly, $N^{2}$ can be written as: 

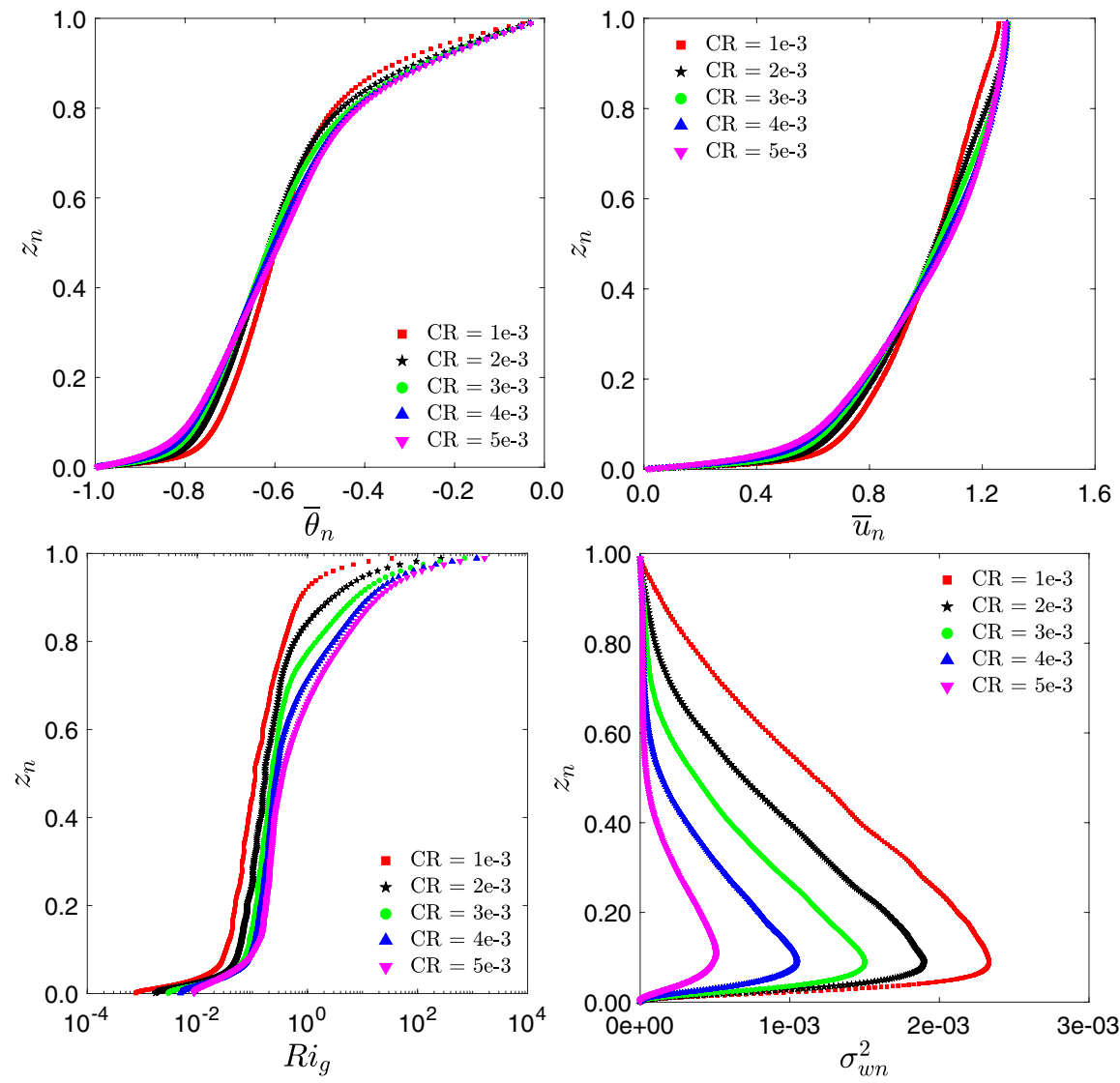

Fig. 8 Vertical profiles of normalized potential temperature (top-left panel), longitudinal velocity (top-right panel), gradient Richardson number (bottom-left panel), and vertical velocity variance (bottom-right panel). Simulated data from five different DNS runs are represented by different colored symbols in these plots. In the legends, $C R$ represents normalized cooling rates. All the profiles correspond to $T_{n}=100$

$$
N^{2}=R i_{b}\left(\frac{U_{b}^{2}}{h^{2}}\right)\left(\frac{\partial \bar{\theta}_{n}}{\partial z_{n}}\right) .
$$

The velocity variances, TKE, and temperature variance can be normalized as:

$$
\begin{gathered}
\sigma_{u_{n}}^{2}=\frac{\sigma_{u}^{2}}{U_{b}^{2}}, \\
\sigma_{v_{n}}^{2}=\frac{\sigma_{v}^{2}}{U_{b}^{2}},
\end{gathered}
$$



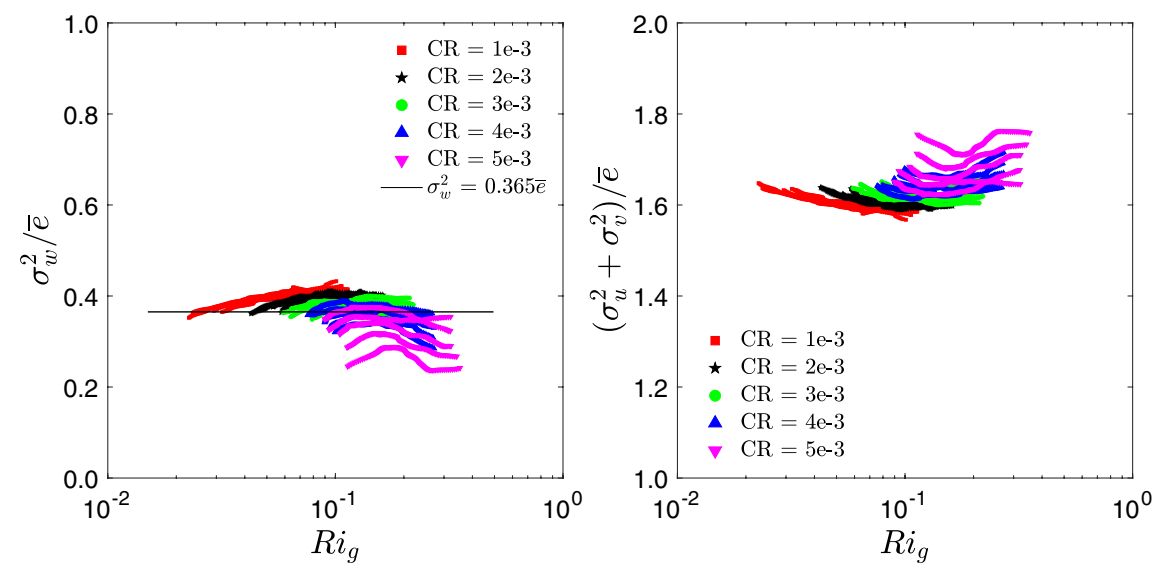

Fig. 9 Dependence of vertical and horizontal velocity variances on gradient Richardson number. The variances are normalized by TKE. Simulated data from five different DNS runs are represented by different colored symbols in these plots. In the legends, $C R$ represents normalized cooling rates

$$
\begin{gathered}
\sigma_{w_{n}}^{2}=\frac{\sigma_{w}^{2}}{U_{b}^{2}}, \\
\bar{e}_{n}=\frac{\bar{e}}{U_{b}^{2}}, \\
\sigma_{\theta_{n}}^{2}=\frac{\sigma_{\theta}^{2}}{\left(\Theta_{t o p}-\Theta_{b o t}\right)^{2}} .
\end{gathered}
$$

Following the above normalization approach, we can also derive the following relationships for the dissipation rate of TKE and variance of temperature fluctuations:

$$
\begin{gathered}
\bar{\varepsilon}=v\left(\frac{U_{b}}{h}\right)^{2} \bar{\varepsilon}_{n}, \\
\bar{\chi}=k\left(\frac{\Theta_{t o p}-\Theta_{b o t}}{h}\right)^{2} \bar{\chi}_{n} .
\end{gathered}
$$

We can combine Eqs. 29d, 29e, 30a, and 30b, we can re-write Eq. 7 as follows:

$$
\bar{\chi}_{n}=\left(\frac{v}{k}\right)\left(\frac{\sigma_{\theta_{n}}^{2}}{\bar{e}_{n}}\right) \bar{\varepsilon}_{n}=\operatorname{Pr}\left(\frac{\sigma_{\theta_{n}}^{2}}{\bar{e}_{n}}\right) \bar{\varepsilon}_{n} .
$$

In a similar fashion, we can utilize Eqs. 25c, 25d, 29d, and 30b to re-write Eq. 16a as follows: 

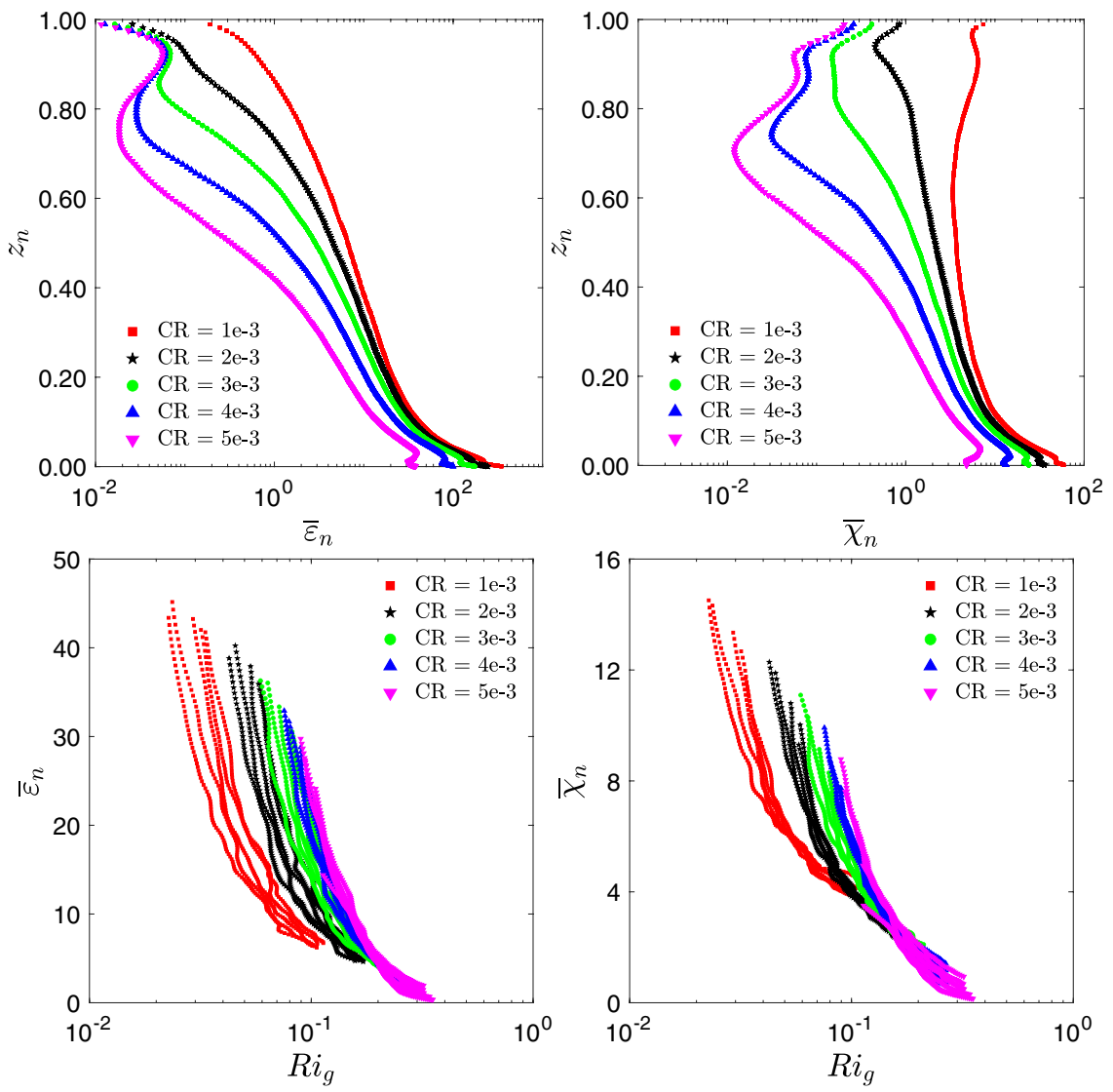

Fig. 10 Vertical profiles of normalized energy dissipation rate (top-left panel) and normalized dissipation of temperature variance (top right panel). All the profiles correspond to $T_{n}=100$. Bottom panel: dependence of the dissipation rates on gradient Richardson number. Simulated data from five different DNS runs are represented by different colored symbols in these plots. In the legends, $C R$ represents normalized cooling rates

$$
\bar{\chi}_{n}=\left(\frac{v}{k}\right)\left(\frac{U_{b} h}{v}\right)\left(\frac{\bar{e}_{n}}{S_{n}}\right)\left(\frac{\partial \bar{\theta}_{n}}{\partial z_{n}}\right)^{2}=\operatorname{PrRe}_{b}\left(\frac{\bar{e}_{n}}{S_{n}}\right)\left(\frac{\partial \bar{\theta}_{n}}{\partial z_{n}}\right)^{2} .
$$

\section{Appendix 3: Supplementary analyses of DNS-generated data}

In Fig. 8, vertical profiles of several key variables are plotted. It is clear that stability monotonically increases with height. As a result, turbulence in the upper part of the domain becomes quasi-laminar (especially for the runs with higher cooling rates).

For continuously turbulent stable boundary layers (SBLs), it has been frequently observed that $R i_{g}$ stays below 0.2 within the SBL (e.g., [9, 26, 45]). Above the SBL, in the 
free atmosphere, $R i_{g}$ becomes much larger. Similar behavior is noticeable in Fig. 8 (bottom-left panel).

We would like to point out that our DNS results are also in agreement with the celebrated 'local scaling' hypothesis by Nieuwstadt [45]. By utilizing the observational data from the Cabauw tower, Nieuwstadt [45] showed that normalized variances remain more or less constant for a wide range of stability conditions. Basu and Porté-Agel [9] analyzed datasets from field campaigns, wind tunnel, and large-eddy simulations and confirmed the original findings of Nieuwstadt. In Fig. 9, normalized variances from our DNS runs are shown.

Recently, Basu et al. [10] found that for $0<R i_{g}<0.2, \bar{\varepsilon}=0.23 \bar{e} S$ and $\bar{\varepsilon}=0.63 \sigma_{w}^{2} S$. Thus, one can easily deduce that $\sigma_{w}^{2} / \bar{e}=0.365$. This relationship is overlaid on the DNS data in the left panel of Fig. 9. Except for the data from the simulation with the highest cooling rate, this relationship is reasonably valid. Based on the LES data, [9] reported: $\sigma_{w}^{2} / \bar{e}=0.39$. Our DNS-based result is remarkably close to this previous finding.

The vertical profiles of dissipation rates are shown in the top panel of Fig. 10. As expected, the dissipation rates decrease with increasing height. For $z / h<0.1$, due to the viscous effects, the values of the dissipation rates are very high. Thus, for the computations of various length scales, we disregarded data from this region.

In our DNS runs, the bulk Reynolds number $\left(R e_{b}\right)$ is fixed along with the initial turbulence level. Given this setup, the production of shear-generated turbulence remains the same across all the simulations. However, the destruction of turbulence due to the buoyancy effects is more predominant for the runs with higher imposed cooling rates. As a result, under steady-state condition, the dissipation rates are lower in the simulations with higher cooling rates. Such behavior can be clearly seen in both the top and bottom panels of Fig. 10.

\section{References}

1. Abe H, Antonia RA (2011) Scaling of normalized mean energy and scalar dissipation rates in a turbulent channel flow. Phys Fluids 23(055):104

2. Abe H, Antonia RA, Kawamura H (2009) Correlation between small-scale velocity and scalar fluctuations in a turbulent channel flow. J Fluid Mech 627:1-32

3. Alam S, Guha A, Verma MK (2019) Revisiting Bolgiano-Obukhov scaling for moderately stably stratified turbulence. J Fluid Mech 875:961-973

4. Anderson PS (2009) Measurement of Prandtl number as a function of Richardson number avoiding self-correlation. Boundary-Layer Meteorol 131:345-362

5. Antonia RA, Chambers AJ (1980) On the correlation between turbulent velocity and temperature derivatives in the atmospheric surface layer. Boundary-Layer Meteorol 18:399-410

6. Antonia RA, Van Atta CW (1975) On the correlation between temperature and velocity dissipation fields in a heated turbulent jet. J Fluid Mech 67:273-288

7. Antonia RA, Zhou T, Xu G (2001) Correlation between energy and temperature dissipation rates in turbulent flows. In: IUTAM symposium on geometry and statistics of turbulence, pp 185-190

8. Basu S (2015) A simple approach for estimating the refractive index structure parameter $\left(c_{n}^{2}\right)$ profile in the atmosphere. Opt Lett 40:4130-4133

9. Basu S, Porté-Agel F (2006) Large-eddy simulation of stably stratified atmospheric boundary layer turbulence: a scale-dependent dynamic modeling approach. J Atmos Sci 63:2074-2091

10. Basu S, He P, DeMarco AW (2020) Parametrizing the energy dissipation rate in stably stratified flows. Boundary-Layer Meteorol. https://doi.org/10.1007/s10546-020-00559-0

11. Béguier C, Dekeyser I, Launder BE (1978) Ratio of scalar and velocity dissipation time scales in shear flow turbulence. Phys Fluids 21:307-310

12. Boffetta G, De Lillo F, Mazzino A, Musacchio S (2012) Bolgiano scale in confined Rayleigh-Taylor turbulence. J Fluid Mech 690:426-440 
13. Bolgiano R Jr (1959) Turbulent spectra in a stably stratified atmosphere. J Geophys Res 64:2226-2229

14. Bolgiano R Jr (1962) Structure of turbulence in stratified media. J Geophys Res 67:3015-3023

15. Cheng Y, Canuto VM, Howard AM (2002) An improved model for the turbulent PBL. J Atmos Sci 59:1550-1565

16. Chung D, Matheou G (2012) Direct numerical simulation of stationary homogeneous stratified sheared turbulence. J Fluid Mech 696:434-467

17. Corrsin S (1958) Local isotropy in turbulent shear flow. Technical report NACA RM 58B11. National Advisory Committee for Aeronautics

18. Coulman CE, Vernin J, Coqueugniot Y, Caccia JL (1988) Outer scale of turbulence appropriate to modeling refractive-index structure profiles. Appl Opt 27:155-160

19. Dewan EM, Good RE, Beland R, Brown J (1993) A model for $C_{n}^{2}$ (optical turbulence) profiles using radiosonde data. Technical report PL-TR-93-2043, Environmental Research Papers, No. 1121

20. Dougherty JP (1961) The anisotropy of turbulence at the meteor level. J Atmos Terr Phys 21:210-213

21. Efron B (1982) The jackknife, the bootstrap, and other resampling plans, vol 38. SIAM, New York

22. Elghobashi SE, Launder BE (1983) Turbulent time scales and the dissipation rate of temperature variance in the thermal mixing layer. Phys Fluids 26:2415-2419

23. Ellison TH (1957) Turbulent transport of heat and momentum from an infinite rough plane. J Fluid Mech 2:456-466

24. Fulachier L, Antonia RA (1984) Spectral analogy between temperature and velocity fluctuations in several turbulent flows. Int J Heat Mass Transf 27:987-997

25. Fulachier L, Dumas R (1976) Spectral analogy between temperature and velocity fluctuations in a turbulent boundary layer. J Fluid Mech 77:257-277

26. Garratt JR (1982) Observations in the nocturnal boundary layer. Boundary-Layer Meteorol 22:21-48

27. Gavrilov NM, Luce H, Crochet M, Dalaudier F, Fukao S (2005) Turbulence parameter estimations from high-resolution balloon temperature measurements of the MUTSI-2000 campaign. Ann Geophys 23:2401-2413

28. Grisogono B (2010) Generalizing 'z-less' mixing length for stable boundary layers. Q J R Meteorol Soc 136:213-221

29. Hao Z, Zhou T, Chua LP, Yu SCM (2008) Approximations to energy and temperature dissipation rates in the far field of a cylinder wake. Exp Thermal Fluid Sci 32:791-799

30. He P (2016) A high order finite difference solver for massively parallel simulations of stably stratified turbulent channel flows. Comput Fluids 127:161-173

31. He P, Basu S (2016) Extending a surface-layer $c_{n}^{2}$ model for strongly stratified conditions utilizing a numerically generated turbulence dataset. Opt Express 24:9574-9582

32. Hunt J, Moin P, Lee M, Moser RD, Spalart P, Mansour NN, Kaimal JC, Gaynor E (1989) Cross correlation and length scales in turbulent flows near surfaces. In: Fernholz HH, Fiedler HE (eds) Advances in turbulence, vol 2. Springer, Berlin, pp 128-134

33. Hunt JCR, Stretch DD, Britter RE (1988) Length scales in stably stratified turbulent flows and their use in turbulence models. In: Puttock JS (ed) Stably stratified flow and dense gas dispersion. Clarendon Press, Oxford, pp 285-321

34. Itsweire EC, Koseff JR, Briggs DA, Ferziger JH (1993) Turbulence in stratified shear flows: implications for interpreting shear-induced mixing in the ocean. J Phys Ocean 23:1508-1522

35. Kantha L, Luce H (2018) Mixing coefficient in stably stratified flows. J Phys Ocean 48:2649-2665

36. Kantha LH, Clayson CA (1994) An improved mixed layer model for geophysical applications. J Geophys Res 99(C12):25235-25266

37. Kumar A, Chatterjee AG, Verma MK (2014) Energy spectrum of buoyancy-driven turbulence. Phys Rev E 90(023):016

38. Li D (2019) Turbulent Prandtl number in the atmospheric boundary layer-Where are we now? Atmos Res 216:86-105

39. Mater BD, Schaad SM, Venayagamoorthy SK (2013) Relevance of the Thorpe length scale in stably stratified turbulence. Phys Fluids 25(076):604

40. Mellor GL, Yamada T (1982) Development of a turbulence closure model for geophysical fluid problems. Rev Geophys 20:851-875

41. Monin AS, Ozmidov RV (1985) Turbulence in the ocean. D. Reidel Publishing Company, Dordrecht, p 247

42. Mooney CF, Duval RD (1993) Bootstrapping: a nonparametric approach to statistical inference, vol 95. Sage, London, p 73 
43. Muschinski A (2015) Temperature variance dissipation equation and its relevance for optical turbulence modeling. J Opt Soc Am A 32:2195-2200

44. Niemela JJ, Skrbek L, Sreenivasan KR, Donnelly RJ (2000) Turbulent convection at very high Rayleigh numbers. Nature 404:837

45. Nieuwstadt FTM (1984) The turbulent structure of the stable, nocturnal boundary layer. J Atmos Sci 41:2202-2216

46. Obukhov AM (1959) On influence of buoyancy forces on the structure of temperature field in a turbulent flow. Dokl Akad Nauk SSSR 125:1246-1248

47. Ozmidov RV (1965) On the turbulent exchange in a stably stratified ocean. Izv Acad Sci USSR Atmos Ocean Phys 1:853-860

48. Panchev S (1975) On the existence of power-law relationships in oceanic turbulence spectra. Atm Ocean Phys 11:381-383

49. Pope SB (2000) Turbulent flows. Cambridge University Press, Cambridge, p 771

50. Prasad RR, Meneveau C, Sreenivasan KR (1988) Multifractal nature of the dissipation field of passive scalars in fully turbulent flows. Phys Rev Lett 61:74

51. Rosenberg D, Pouquet A, Marino R, Mininni PD (2015) Evidence for Bolgiano-Obukhov scaling in rotating stratified turbulence using high-resolution direct numerical simulations. Phys Fluids 27(055): 105

52. Schmitt F, Schertzer D, Lovejoy S, Brunet Y (1996) Multifractal temperature and flux of temperature variance in fully developed turbulence. Europhys Lett 34:195

53. Smyth WD, Moum JN (2000) Length scales of turbulence in stably stratified mixing layers. Phys Fluids $12: 1327-1342$

54. Sreenivasan KR, Antonia RA (1997) The phenomenology of small-scale turbulence. Ann Rev Fluid Mech 29:435-472

55. Sukoriansky S, Galperin B, Perov V (2006) A quasi-normal scale elimination model of turbulence and its application to stably stratified flows. Nonlinear Proc Geophys 13:9-22

56. Tatarski VI (1961) Wave propagation in a turbulent medium. McGraw-Hill, London, p 285

57. Tatarskii VI (1971) The effects of the turbulent atmosphere on wave propagation. Israel Program for Scientific Translations, Jerusalem, p 472

58. Taylor GI (1935) Statistical theory of turbulence. Proc R Soc Ser A 151:421-444

59. Tennekes H, Lumley JL (1972) A first course in turbulence. The MIT Press, London, p 300

60. Thorpe SA (1977) Turbulence and mixing in a Scottish loch. Philos Trans R Soc Lond A 286:125-181

61. Thorpe SA (2005) The turbulent ocean. Cambridge University Press, Cambridge, p 439

62. VanZandt TE, Green JL, Gage KS, Clark WL (1978) Vertical profiles of refractivity turbulence structure constant: comparison of observations by the Sunset radar with a new theoretical model. Radio Sci 13:819-829

63. Verma MK (2018) Physics of buoyant flows. World Scientific, Singapore, p 352

64. Wyngaard JC (1973) On surface-layer turbulence. In: Haugen DA (ed) Workshop on micrometeorology. Amer. Meteorol. Soc., New York, pp 101-149

65. Wyngaard JC (2010) Turbulence in the atmosphere. Cambridge University Press, Cambridge, p 393

66. Wyngaard JC, Coté OR (1971) The budgets of turbulent kinetic energy and temperature variance in the atmospheric surface layer. J Atmos Sci 28:190-201

67. Wyngaard JC, Izumi Y, Collins SA (1971) Behavior of the refractive-index-structure parameter near the ground. J Opt Soc Am 61:1646-1650

68. Xu G, Antonia RA, Rajagopalan S (2000) Scaling of mean temperature dissipation rate. Phys Fluids 12:3090-3093

69. Zhou T, Antonia RA (2000) Approximations for turbulent energy and temperature variance dissipation rates in grid turbulence. Phys Fluids 12:335-344

Publisher's Note Springer Nature remains neutral with regard to jurisdictional claims in published maps and institutional affiliations. 